\title{
Measurement properties of simple biomechanical measures of walking effort
}

\author{
Michael Raymond Pierrynowski*
}

\author{
School of Rehabilitation Science, McMaster University, Hamilton, ON, Canada
}

(Received 21 December 2007; final version received 21 July 2008)

\begin{abstract}
A simple measure of overall walking effort would be valuable to patients and clinicians to select suitable treatment interventions and to monitor progress. In this paper, the reproducibility and responsiveness of seven potentially useful clinical measures of walking effort are presented. These walking effort outcomes were derived from a compass gait model and space curve displacement, acceleration and differential geometry theory. The walking effort outcomes were primarily calculated from the motion of a point on or in the rigid body pelvis as a patient walked cyclically. These motion data were collected from eight healthy volunteers who each walked on a treadmill for $4 \mathrm{~min}$, at four different speeds, repeated twice. Four of the seven walking effort outcomes clearly had better measurement properties. The path length ratio, acceleration ratio, Frenet-Serret torsion and Frenet-Serret energy had excellent reproducibility (ICC $>0.8$ ) and responded to a small change in walking speed $(<0.03 \mathrm{~m} / \mathrm{s})$ compared to two versions of the biomechanical efficiency quotient and the Frenet-Serret curvature. The measurement properties of most outcomes were not consistently improved using a point in versus on the pelvis. This study presents four biomechanical walking effort outcomes that have good theoretical underpinnings, excellent reproducibility and responsiveness, are simple and easy to administer with relatively inexpensive equipment, and can be used in real world environments. However, future work must investigate the minimal clinically important change of these outcome measures before they can be used in clinical practice.
\end{abstract}

Keywords: walking effort; measurement properties; space curve; differential geometry (53A-xx)

\section{Introduction}

Objective measures of walking ability exist primarily in motion capture laboratories and typically use sophisticated equipment that quantitatively measures the kinematics and kinetics of the segments and joints of a patient. Although these assessments provide valuable detailed information a clinician often wants a simple measure of the overall walking effort to monitor patient progress and select suitable treatment interventions [6]. In this paper, the theory and measurement properties of seven potentially useful clinical measures of walking effort are discussed. These biomechanical walking effort outcomes are all designed to be clinically useful, that is, they are clinically feasible (simple to collect using relatively inexpensive equipment) and they objectively quantify small differences in walking effort.

A biomechanical measure of walking effort, based on a model of compass walking and its determinants, has been proposed $[13,14]$. Their biomechanical efficiency quotient (BEQ) requires independent measures of the average stride length (SL) and the vertical

*Email: pierryn@mcmaster.ca 
displacement of a point on the sacrum during walking and standing sacrum height. The reliability of the BEQ was not reported $[13,14]$, but they do provide data that demonstrate that the vertical displacement of the sacrum is linearly associated with oxygen consumption $(r=0.9)$, suggesting that this component of the BEQ calculation has construct validity. Additionally, they report that the BEQ could discriminate patients wearing different foot orthoses [14]. The BEQ supports the concept that a sensor which measures the time-varying displacement of a single point on a patient may provide a simple measure of walking effort. However, the BEQ does not consider the lateral and forward-backward body shifts or the motion of the sacral point relative to the motion of the patient's mass centre.

As a first step to determine if a biomechanical walking effort outcome is clinically useful, it is helpful to examine its measurement properties. Seven criteria should be considered as suggested by the Scientific Advisory Committee of the Medical Outcomes Trust [17]. These criteria include: content, criterion and construct validities, reproducibility, floor or ceiling effects, responsiveness and interpretability. These criteria are defined and applied to a biomechanical measure of walking effort in Table 1 . The purpose of this paper is to present the theoretical underpinnings and some of the measurement properties of the BEQ and six new biomechanical measures of walking effort.

\section{Background}

This section provides a concise summary and notation of mathematical representations of the motion of points and rigid bodies. These concepts are used to define seven biomechanical measures of walking effort.

\subsection{General considerations}

Normal human walking is typically defined as a sequence of closely identical repetitive body motions. In this paper, the walking effort outcomes all require a set of cyclical patterns. Defining an easily identified cyclical event within a continuous walk (i.e. the initial contact of the right or left foot with the ground) one can partition a long series of kinematic data into a set of small cyclical sequences. If the kinematic data is the motion of a point on the foot/sacrum, one can define a SL for each walking cycle as the displacement of the foot/sacrum point from the start to end of each walking cycle.

An estimate of average SL is the mean of multiple measures. The confidence in this average value increases with the ever larger number of strides. However, occasionally, humans execute a short or long stride within a sequence of relatively constant length strides [11]. It is possible that a more robust estimate of average SL, the median, will minimize the influence of the occasionally present too long or too short stride. Therefore, in this paper both mean and median statistics are used to provide average estimates.

The three-dimensional $\left(\mathcal{R}^{3}\right)$ motion of a point on a rigid body traces a space curve. If time is defined to fall in the interval [0,1], the space curve's notation is $\mathcal{R}^{3} \times[0,1]$. The pose of a rigid object in $\mathcal{R}^{3}$ relative to a reference position is $\operatorname{SE}(3)$, the special Euclidean group in $\mathcal{R}^{3}$, which includes both orientation and translation. In biomechanics, $\mathrm{SE}(3) \times[0,1]$ is measured using kinematic data acquisition devices which quantify the space curves of three or more non-collinear points on a rigid body [19]. One notes that the calibration of a kinematic data acquisition device defines both signed directions and an origin that are mirrored within the definition of the rigid body. Therefore, $\operatorname{SE}(3) \times[0,1]$ defines the relative (reference to pose) orientation and position of a rigid body.

The position of a rigid body is often defined as the location of the centroid of three or more non-collinear markers placed on that body [19]. However, the space curve traced 


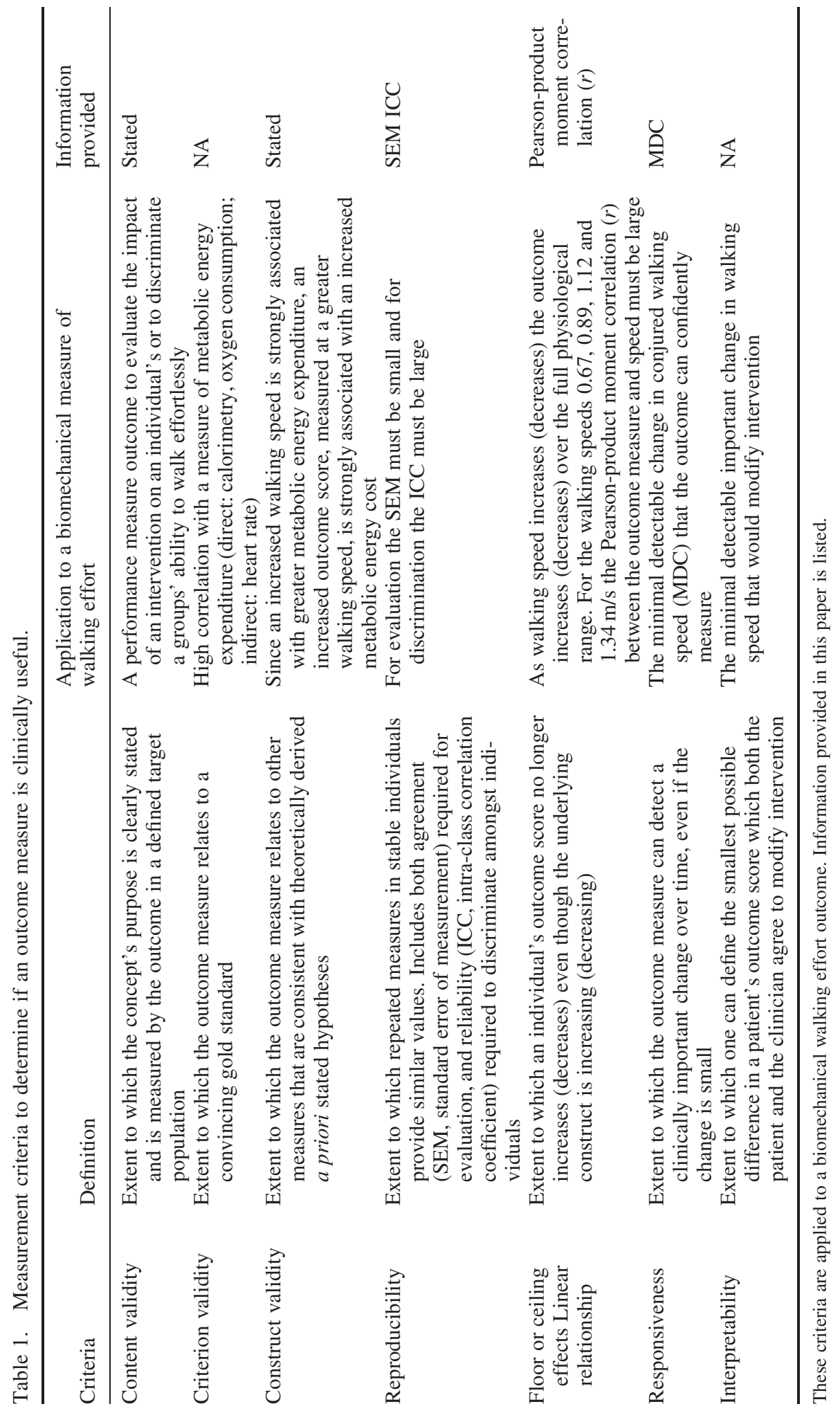


by this point (compared to some other point on the rigid body) depends on the rotation and translation motions of the rigid body. This space curve can be of greater length than some other point on or within the rigid body. As an example, consider a sphere rolling on a plane, the motion of any point on the surface of the sphere traces a space curve of greater length than the space curve traced by the sphere's centre. Newtonian mechanics dictates that the work required moving the rigid body has translational and rotational components. For a walking human, the translational work component is greater that the rotational work expended [22]. In this paper a point in the pelvis was modelled to mimic the centre of mass motion of a walking human. For each volunteer this 'in the pelvis' point was assumed to have a fixed position relative to the 'on the sacrum' specific point.

As will be shown, it is sometimes convenient to extract unique characteristics of the space curve independent of its orientation and location in $\mathcal{R}^{3}$. Assume that the space curve's displacement-time profile $\left(\mathbf{r}=\mathcal{R}^{3} \times[0,1]\right)$ can be consecutively differentiated to provide its velocity $\left(\mathbf{r}^{\prime}\right)$, acceleration $\left(\mathbf{r}^{\prime \prime}\right)$ and jerk $\left(\mathbf{r}^{\prime \prime \prime}\right)$. The resultant acceleration-time profile $\left(a_{\mathrm{r}}=\left|\mathbf{r}^{\prime \prime}\right|\right.$, where $|\cdot|$ is the magnitude) can be expressed in tangential $\left(a_{\mathrm{t}}=\mathbf{r}^{\prime} \cdot \mathbf{r}^{\prime \prime} /\left|\mathbf{r}^{\prime}\right|\right)$ and normal $\left(a_{\mathrm{n}}=\left|\mathbf{r}^{\prime} \times \mathbf{r}^{\prime \prime}\right| /\left|\mathbf{r}^{\prime}\right|\right)$ components. These two orthogonal acceleration components act on the space curve forcing it to curve away from linearity and planarity.

At present, this paper has considered space curves measured at equal time periods since kinematic data acquisition systems almost always use this convention. However, one can consider a non-uniform time function such that the velocity of a point tracing the space curve is constant (i.e. moving equivalent arc length distances in each non-uniform time period). An arc length parameterized space curve (s) has zero acceleration and jerk profiles. More importantly, s's deviation from linearity and planarity can be defined as curvature $\left(\kappa=\left|\mathbf{s}^{\prime} \times \mathbf{s}^{\prime \prime}\right| /\left|\mathbf{s}^{\prime}\right|^{3}\right)$ and torsion $\left(\tau=\left(\mathbf{s}^{\prime} \times \mathbf{s}^{\prime \prime}\right) \cdot \mathbf{s}^{\prime \prime \prime} /\left|\mathbf{s}^{\prime} \times \mathbf{s}^{\prime \prime}\right|^{2}\right)$ which are independent of the space curve's $(\mathbf{r})$ orientation and position in global $\mathcal{R}^{3}$ [1]. Additionally, three unit length orthogonal vectors, the tangential $\left(\mathbf{T}=\mathbf{s}^{\prime} /|\mathbf{s}|\right)$, normal $\left(\mathbf{N}=\mathbf{T} /\left|\mathbf{T}^{\prime}\right|\right)$ and binormal $(\mathbf{B}=\mathbf{T} \times \mathbf{N})$ vectors, define a local frame of reference attached to a moving point on $\mathbf{s}$. The set $\{\kappa, \tau, \mathbf{T}, \mathbf{N}$ and $\mathbf{B}\}$, and their derivatives, are related using the Frenet-Serret formulae. These formulae are of fundamental importance in differential geometry [4]. Some differential geometry proofs will be used below to define clinically useful walking effort outcomes.

\subsection{Walking effort using a compass model (BEQ, BEQp)}

The SL and the measured vertical displacement of the pelvis $(m)$ during walking, and the standing pelvis height $(h)$ are required to estimate the BEQ. Specifically, the BEQ $=m / p$, where $p=$ predicted vertical pelvis displacement $=\frac{1}{2}\left(h-\sqrt{ }\left(h^{2}-\left(\frac{1}{4} \mathrm{SL}\right)^{2}\right)\right)$ which estimates the vertical motion of the pelvis as if the legs were rigid rods over which the person vaults (i.e. compass walking) [14]. BEQ values near one indicate that the person is walking with least effort. In this paper, the motion of a point on the foot and on the pelvis were used to measure two SLs which were then used to calculate BEQ and BEQp, respectively. BEQp was defined to determine if BEQ could be measured without using dynamic foot data thereby further simplifying kinematic data acquisition.

\subsection{Walking effort using space curve displacement (PLR)}

The path length of a point on or within the pelvis (displacement) divided by its direct path (distance) defines the path length ratio (PLR). The PLR assumes undulating paths require more effort than direct paths to travel from A to B. 


\subsection{Walking effort using space curve acceleration (ALR)}

The ratio of the sum of the squared resultant accelerations $\left(\sum a_{\mathrm{r}}^{2}\right)$ and the sum of the squared tangential accelerations $\left(\sum a_{\mathrm{t}}^{2}\right)$ defines the acceleration ratio (ALR). The ALR penalizes motion along curves with large $a_{\mathrm{r}}$. Tracing a space curve with minimal $\sum a_{\mathrm{r}}^{2}$ has been proved to minimize the total energy cost [2].

\subsection{Walking effort using differential geometry (FSC, FST, FSE)}

Consider the arc length parameterized space curve(s) defined by the motion of a point on or in a person's pelvis during one walking cycle. Although the pelvis is displaced forward during typical overground level walking, one can remove this constant forward motion component. This manipulated data is akin to that obtained as if the volunteer walked on a constant speed treadmill and returns to the same body configuration at the start (end) of each walking cycle. One now observes that $\mathbf{s}$ forms a closed loop and that the $\mathbf{T}, \mathbf{N}$ and $\mathbf{B}$ local orientation vectors, tracing $\mathbf{s}$, each trace closed paths on a unit sphere $\left(\mathbf{S}^{2}\right)$ since they are unit length vectors. These paths are the tangent, normal and binormal indicatrices. From differential geometry [1] it has been proved that the length of the tangent indicatrix must be greater than or equal to $2 \pi$ where length equals $2 \pi$ defines a great circle path on $\mathbf{S}^{2}$ (Frechel's Theorem). Since the integral (sum) of the curvatures $(\kappa)$ of a closed space curve equals the length of the tangent indicatrix, the first differential geometry outcome was defined as Frenet-Serrat curvature $(\mathrm{FSC})=1 /(2 \pi) \sum \kappa$. In a similar vein, the length of the binormal indicatrix equals the integral (sum) of the absolute values of the torsions $(|\tau|)$ which can be interpreted as $2 \pi$ minus the area enclosed by the tangent indicatrix. Comparing this sum to the area of a unit sphere $(4 \pi)$ the second differential geometry outcome was defined as Frenet-Serrat torsion $(\mathrm{FST})=\left(2 \pi-\sum|\tau|\right) /(4 \pi)+1$, where the one is added to force FST to be similar to the other outcomes (i.e. greater than or equal to one). Both FSC and FST have the property that values greater than one indicate space curves that are not respectively linear or planar, and are longer than more direct paths, thereby indicating more effort motion paths. The third differential geometry outcome considers the sum of the squared curvatures (plus one to make it similar to the other outcomes) (Frenet-Serrat energy (FSE) $\left.=\sum \kappa^{2}+1\right)$. FSE (minus one) has been labelled the 'energy integral' [15] since it quantifies the amount of additional energy needed to stretch a spring around a sphere whose path deviates from a (great) circular path. In this regard the portions of an arc length parameterized space curve most deviated from a direct linear and planar path has the greatest influence on the additional energy required. It is the squared magnitude of the path deviation that is associated with more effort motion paths.

\section{Methods}

Eight volunteers (four males and four females) were selected from a convenience sample of university students and faculty. Inclusion criteria included asymptomatic healthy individuals; volunteers with a history of cardiovascular, pulmonary, neurological or orthopaedic diseases were excluded. The average age, height and mass of the volunteers were 27.8 (24-50) yr, $173(155-196) \mathrm{cm}$ and 75 (45-122) $\mathrm{kg}$, respectively. This study was approved by a combined University and Hospital Ethics Review Board.

Following informed consent, and a familiarization period walking on the treadmill (True S.O.F.T System 500, True Fitness Technology, Inc., O’Fallon, MO, USA), the volunteers walked for $4 \mathrm{~min}$ at each of four different treadmill speeds $(0.67,0.89,1.12$ and $1.34 \mathrm{~m} / \mathrm{s} ; 1.5,2.0,2.5$ and $3.0 \mathrm{mph})$. After a $15 \mathrm{~min}$ break, the protocol was repeated. The order of treadmill speeds, within each block of four, was randomly assigned. 
The motion of a single marker placed on the left heel and four markers placed on the sacrum were recorded using a vertically mounted kinematic data acquisition system (OptoTrak, Northern Digital, Inc, Waterloo, Canada). The middle lens of the camera was positioned approximately $3 \mathrm{~m}$ behind the volunteer and $1 \mathrm{~m}$ above the floor. This device can record the $3 \mathrm{D}$ location of a marker with better than $0.2 \mathrm{~mm}$ accuracy. Data capture was set at 100 frames per second. Prior to the walking trials each volunteer stood quietly, arms at sides, eyes open, for $15 \mathrm{~s}$ whilst the position of the five markers were recorded. These data were used to define the standing sacral height and the 3D position of each marker relative to each volunteer's lateral-medial, anterior-posterior and upward-downward directions. The markers were newly applied for each set of four treadmill walks.

The kinematic data acquisition system measured the space curves for the left heel marker and the four markers on the sacrum whilst each volunteer stood in the reference position and walked on the treadmill. The heel marker's motion during walking was used to define walking cycle boundaries. First, the vertical motion of the heel marker was interpolated and smoothed using a quintic spline (GCVSPL, available from http://www. netlib.org/gcv/gcvspl), then scaled to the interval zero to one. Sequences of frames were then extracted where the smoothed, interpolated and scaled heel marker's motion path was less than 0.15 . Within each of these sequences the time (frame) when the foot marker was at its lowest position was identified. Pilot work demonstrated that this automated method consistently located this event compared to visual inspection. A similar algorithm reliably located cycle boundaries, albeit not heel contact, from the space curve of a sacral marker.

The markers on the sacrum were considered a rigid body, centred at its centroid, and its time varying orientation and position were estimated using an algebraic singular value decomposition technique [19]. Since the motion of a point on the pelvis has two motion components, the motion of a 'point' in the pelvis and the motion of the pelvis about that 'point', an estimate of the location of the 'point' that has minimal motion was calculated. This minimal motion 'point' in the pelvis, relative to the centroid of the markers on the pelvis, was identified using an optimization routine that minimizes a function subject to bounds using a quasi-Newton method and a finite-difference gradient. Specifically, the optimization subroutine DBCONF (IMSL Numerical Library, Visual Numerics, Houston, TX, USA) was used to find the vector from on to in, that minimized the length of the three-dimensional space curve of the in-pelvis point as the volunteer walked. For interest, for one volunteer, the in pelvis point was $11 \mathrm{~mm}$ rightward, $91 \mathrm{~mm}$ forward and $78 \mathrm{~mm}$ downwards from the centre of his sacrum. Since this in-pelvis point approximates the midpoint of the volunteer's hip joints, and this point visually demonstrates smaller motions during gait than the sacrum, the technique was deemed successful to locate a minimal motion point in the pelvis.

For each volunteer $(V=8)$, for each walking speed $(S=4)$, for each trial $(T=2)$, and for every walking cycle ( $C=$ variable), the seven walking effort outcomes $(O=7)$, for the marker on and in the pelvis $(P=2)$, were calculated. The mean and median values $(M=2)$ of the $C$ walking cycles were then calculated. For the 28 combinations of $O, P$ and $M$, a commercial statistical package (SPSS V15.0.1, SPSS, Inc., Chicago, IL, USA) was used to generate the variance components attributed to the $V, S, T$, main effects and the $V S$, $V T$, and $S T$ interactions. The VST 'interaction' was defined to be the residual error and its square root was the standard error of the measure (SEM). Generalizability theory was then used to estimate reliability. Reliability was defined as the agreement of the repeated values [9]. Further, the 95\% confidence intervals around the reliability values were estimated [8].

The literature provides a variety of methods to quantify the responsiveness of an outcome measure to detect meaningful clinical change [20]. In this paper, responsiveness was defined as a conjured change in walking speed not the change in the outcome value. This definition 
allows direct comparison between the various outcomes on the same measurement scale. The conjured change in walking speed transformation is analogous to the same automobile travelling twice at the same speed but with different fuel consumptions. If one considers the fuel consumptions equivalent, one can infer that the vehicle must have travelled at different speeds. In this paper, the response of each outcome was assumed to be linearly associated with walking speed (evaluated using the Pearson product moment correlation) and the slope of the linear regression line was used, along with the SEM, to estimate the minimal detectable conjured change in walking speed. Small values are desired.

\section{Results}

Results demonstrate that several outcomes clearly had better measurement properties. PLR, ALR, FST and FSE, compared to BEQ, BEQp and FSC, all had excellent agreements (ICC > 0.8) (Figure 1). All of the outcomes, except BEQ and PLR, were linearly associated with walking speed (correlations $>0.8$ ) (Figure 2). Lastly, PLR, ALR, FST and FSE were all able to respond to small changes in walking speed $(<0.03 \mathrm{~m} / \mathrm{s})$, whilst BEQ and BEQp performed poorly (Table 2). The measurement properties of the outcomes were not consistently different using median instead of mean statistics, except for BEQ that clearly favoured the median variant. Similarly, the use of a point in versus on the pelvis offered no advantage except for PLR.

\section{Discussion}

The limitations of common clinical tools to measure walking effort led Kerrigan and her colleagues $[13,14]$ to develop a simple measure of walking effort. Their BEQ was successfully used to discriminate patients walking at different speeds, with and without ankle-foot orthoses and knee immobilizing knee braces, and patients with neurological gait impairments. Although, the BEQ was shown to be discriminative $(\mathrm{ICC}=0.7$ ) our results suggest it is not as good as the competing PLR, ALR, FST and FSE outcomes. Our findings also suggest that the BEQ has poor evaluative properties (high SEM) suggesting its limitations as a general purpose clinical tool to measure walking effort.

A clinical measure of walking effort based on heart rate $[5,16]$ is commonly used although its reliability is variable $[3,12,21]$. Although the most valid and reliable method of walking effort is based on the measure of oxygen consumption this technique is not

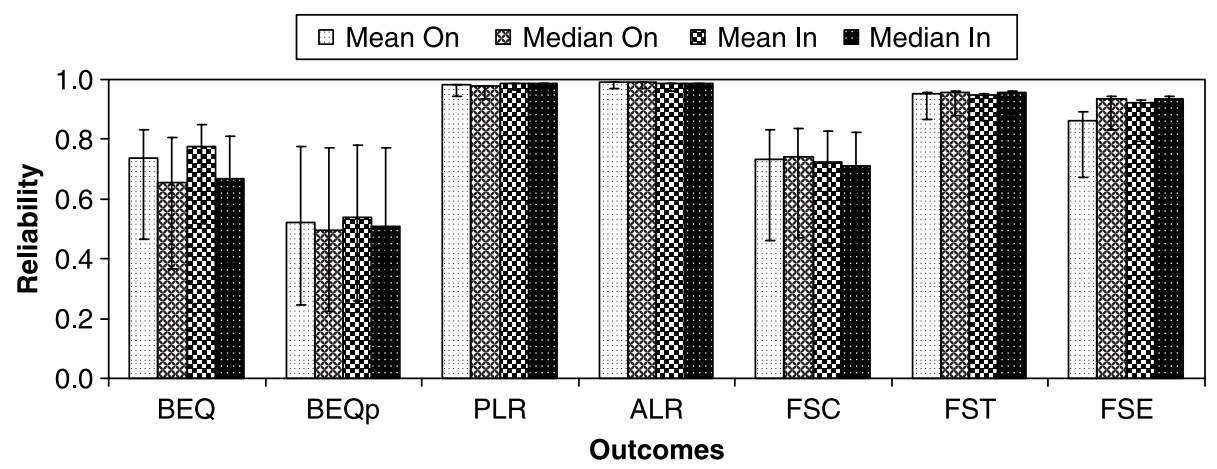

Figure 1. The reliabilities (intra-class coefficients, agreement), and their $95 \%$ confidence intervals, for the four variants of each of the seven walking effort outcome measures. Values near one imply the near perfect agreement of multiple walking cycle measures. Both the mean and median averages of multiple walking cycle estimates were examined. 'On' and 'In' refer to a marker on or in the pelvis (see text for details). 


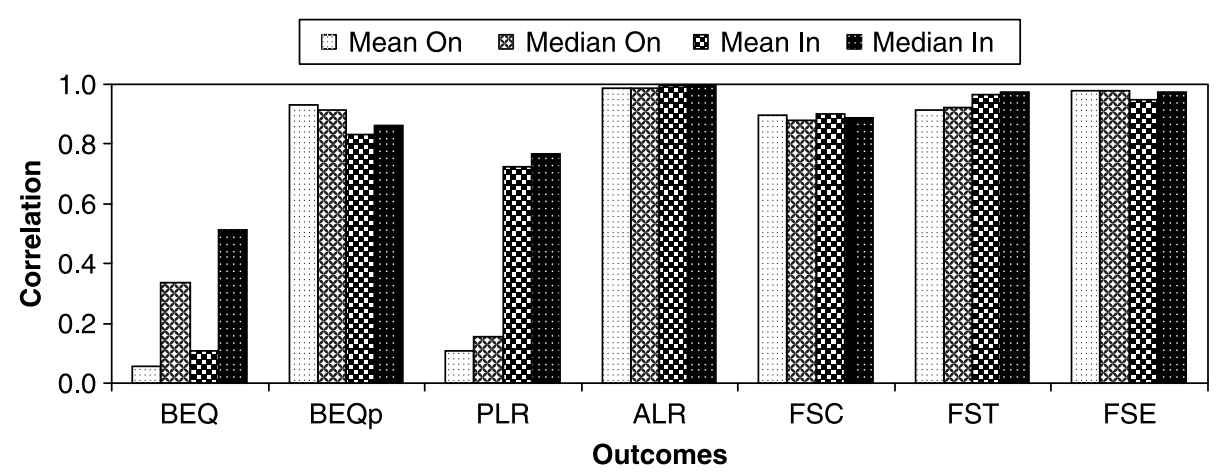

Figure 2. The correlations (Pearson-product moment) between the four variants of each of the seven walking effort outcome measures and walking speed. Values near one imply a near perfect linear relationship. Both the mean and median averages of multiple walking cycle estimates were examined; 'On' and 'In' refer to a marker on or in the pelvis (see text for details).

feasible in the clinical environment. Similarly, mechanical work, power and efficiency measures, derived from data collected in motion analysis laboratories, are not routinely used. Our results demonstrate that there are several simple biomechanical measures of walking effort (PLR, ALR, FST, FSE) that are strongly associated with walking speed, our surrogate measure of walking effort, that can be derived from the motion of a single point placed on the sacrum. However, further work must be completed to specifically examine the association between these measures and gold standard measures. It was also originally thought that the use of a point in the pelvis, compared to a specific point on the sacrum, would provide better measurement properties. Our results to date demonstrate that one only needs to measure the kinematics of a point on the sacrum $\left\{\mathcal{R}^{3} \times[0,1]\right\}$ instead of the pelvis' rigid body motion $\{\mathrm{SE}(3) \times[0,1]\}$ to obtain a biomechanical estimate of walking effort. These data can be inexpensively measured using suitably processed tri-axial accelerometer data instead of more complex $6 \mathrm{df}$ inertial sensors. This has the practical clinical advantage of being less expensive. Finally, these same outcomes were also able to confidently detect a change in walking speed of less than $0.03 \mathrm{~m} / \mathrm{s}$.

Noting that the median is a more robust estimate of the central tendency of an asymmetrical set of values both the mean and median averages were examined to determine if one provides better walking effort measurement properties. The data suggests

Table 2. Selected measurement properties of seven simple biomechanical outcomes to measure walking effort.

\begin{tabular}{lllll}
\hline Outcome & Name & Slope $^{\mathrm{a}}$ & SEM $^{\mathrm{b}, \mathrm{d}}$ & MDC $^{\mathrm{c}, \mathrm{d}}$ \\
\hline BEQ & Biomechanical efficiency quotient & 0.082 & 0.055 & 0.66 \\
BEQp & BEQ using pelvis data & 0.137 & 0.054 & 0.39 \\
PLR & Path length ratio & 0.003 & 0.0001 & 0.03 \\
ALR & Acceleration ratio & 3.166 & 0.030 & 0.01 \\
FSC & Frenet-Serrat curvature & 0.549 & 0.047 & 0.09 \\
FST & Frenet-Serrat torsion & 0.204 & 0.005 & 0.02 \\
FSE & Frenet-Serrat energy & 2.264 & 0.059 & 0.03 \\
\hline
\end{tabular}

Values for the mean average, in the pelvis marker variants are presented.

${ }^{a}$ Slope: change in outcome value over the change in walking speed in $\mathrm{m} / \mathrm{s}$.

${ }^{\mathrm{b}}$ SEM: standard error of measurement, $95 \%$ upper confidence interval value.

${ }^{\mathrm{c}}$ MDC: minimal detectable change in walking speed in $\mathrm{m} / \mathrm{s}, 95 \%$ upper confidence interval value.

${ }^{\mathrm{d}}$ Calculated using the average of 100 walking cycles. 
that the mean value of multiple stride outcomes were equally good as the median value. This may not be surprising since the walking ability of healthy adults were studied, a group who rarely perform atypical steps within a sequence of typical strides [11]. However, in a special population that may demonstrate inconsistent walking patterns (i.e. individuals with cerebral palsy), the use of the median may be useful to obtain better measurement properties. Further work examining the non-normality of multiple stride outcomes in selected special populations is warranted.

This study reports that four walking effort outcomes (PLR, ALR, FST and FSE) had better measurement properties than three others (BEQ, BEQp and FSC). This can be partially explained by noting that BEQ and BEQp only consider the vertical motion of the pelvis and ignore the added information contained within the motions in the lateral and forward-backward directions as influenced by walking speed. A similar argument holds for the poor performance of FSC since it only quantifies changes in pelvis motion curvature within an exemplar planar path. However, changes in the sum of the squared curvatures, which are related to energy requirements, capture an important feature of walking effort. Overall, one must consider the complete space curve motion of the pelvis during walking, as does PLR, ALR and FST, to obtain good estimates of walking effort.

A limitation of the results is that the validity of these outcome measures was not fully addressed. Although the PLR, ALE, FST and FSE outcomes all respond to a change in walking speed, which is consistent with the theoretically derived a priori stated hypotheses that walking speed is strongly associated with walking effort (construct validity), the ability of these outcomes to directly respond to different walking effort's was not tested while a person walks at the same speed. However, recall that ALE and FSE are both mathematically related to energy cost providing additional support for the validity of these outcomes. Finally, some researchers argue that a curvilinear relationship exists between walking speed and effort even though this is not pronounced in the range of walking speeds examined in this paper [10]. Lastly, although walking effort has been defined as a gait pattern that minimizes energy cost [18], its underlying objective may be to minimize pain by reducing mechanical stress or maximize safety by maintaining dynamic stability $[7,23,24]$. Further investigations examining all of these issues are required.

In closing, the PLR, ALE, FST and FSE outcomes were shown to be responsive to relatively small changes in walking speed suggesting that these outcomes can detect small changes in walking speed due to different interventions. Additionally, these biomechanical walking effort outcomes have good theoretical underpinnings, several excellent measurement properties, can be simple and easy to administer with relatively inexpensive equipment, and can be used in real world environments. However, it is unclear what constitutes the minimal change that would influence either a patient's or clinician's behaviour. The interpretability of these outcomes must be further studied.

\section{Acknowledgements}

The author thanks the participants, D. Maltais, M.A. O'Brien and J. Wessel who commented on a previous version of this paper, and the School of Rehabilitation Science that provided financial assistance.

\section{References}

[1] Y. Animov, Differential Geometry and Topology of Curves, CRC Press, Abingdon, 2001.

[2] A.H. Barr, B. Currin, S. Gabriel, and J.F. Hughes, Smooth Interpolation of Orientations with Angular Velocity Constraints using Quaternions, in SIGGRAPH '92: Proceedings of the 19th 
Annual Conference on Computer Graphics and Interactive Techniques, ACM Press, New York, NY, USA, 1992, pp. 313-320.

[3] R. Boyd, S. Fatone, J. Rodda, C. Olesch, R. Starr, E. Cullis, G.R. Nattress, and H.K. Graham, High- or low- technology measurements of energy expenditure in clinical gait analysis? Dev. Med. Child Neurol. 41(10) (1999), pp. 676-682.

[4] W.L. Burke, Applied Differential Geometry, Cambridge University Press, Cambridge, New York, 1985.

[5] P. Butler, M. Engelbrecht, R.E. Major, J.H. Tait, J. Stallard, and J.H. Patrick, Physiological cost index of walking for normal children and its use as an indicator of physical handicap, Dev. Med. Child Neurol. 26(5) (1984), pp. 607-612.

[6] H. Chambers and D.H. Sutherland, A practical guide to gait analysis, J. Am. Acad. Orthop. Surg. 10(3) (2002), p. 222.

[7] R.D. Crowninshield and R.A. Brand, A physiologically based criterion of muscle force prediction in locomotion, J. Biomech. 14(11) (1981), pp. 793-801.

[8] M. Eliasziw, S.L. Young, M.G. Woodbury, and K. Fryday-Field, Statistical methodology for the concurrent assessment of interrater and intrarater reliability: Using goniometric measurements as an example, Phys. Ther. 74(8) (1994), pp. 777-788.

[9] W.J. Evans, C.G. Cayten, and P.A. Green, Determining the generalizability of rating scales in clinical settings, Med. Care 19(12) (1981), pp. 1211-1220.

[10] C. Hall, A. Figueroa, B. Fernhall, and J.A. Kanaley, Energy expenditure of walking and running: comparison with prediction equations, Med. Sci. Sports Exerc. 36(12) (2004), pp. $2128-2134$.

[11] K. Jordan, J.H. Challis, and K.M. Newell, Walking speed influences on gait cycle variability, Gait Posture 26(1) (2007), pp. 128-134.

[12] D.J. Keefer, T. Wayland, J.L. Caputo, K. Apperson, S. McGreal, and D.W. Morgan, Withinand between-day stability of treadmill walking VO2 in children with hemiplegic cerebral palsy, stability of walking VO2 in children with CP, Gait Posture 22(3) (2005), pp. 177-181.

[13] D.C. Kerrigan, M.A. Thirunarayan, L.R. Sheffler, T.A. Ribaudo, and P.J. Corcoran, A tool to assess biomechanical gait efficiency; a preliminary clinical study, Am. J. Phys. Med. Rehabil. 75(1) (1996), pp. 3-8.

[14] D.C. Kerrigan, B.E. Viramontes, P.J. Corcoran, and P.J. LaRaia, Measured versus predicted vertical displacement of the sacrum during gait as a tool to measure biomechanical gait performance, Am. J. Phys. Med. Rehabil. 74(1) (1995), pp. 3-8.

[15] S. Murugesh and M. Lakshmanan, Nonlinear dynamics of moving curves and surfaces: applications to physical systems, Int. J. Bifurcat. Chaos 15(1) (2005), pp. 51-64.

[16] J. Rose, H.J. Ralson, and J.G. Gamble, Energetics of Walking, in Human Walking, J. Rose and J.G. Gamble, eds., 2nd ed., Wiliams and Wilkins, Maryland, 1994, pp. 65-70.

[17] Scientific Advisory Committee of the Medical Outcomes Trust Assessing health status and quality-of-life instruments: attributes and review criteria, Qual. Life Res. 11(3) (2002), pp. 193-205.

[18] M.D. Sockol, D.A. Raichlen, and H. Pontzer, Chimpanzee locomotor energetics and the origin of human bipedalism, Proc. Natl Acad. Sci. USA 104(30) (2007), pp. 12265-12269.

[19] I. Soderkvist and P.A. Wedin, Determining the movements of the skeleton using wellconfigured markers, J. Biomech. 26(12) (1993), pp. 1473-1477.

[20] C.B. Terwee, F.W. Dekker, W.M. Wiersinga, M.F. Prummel, and P.M.M. Bossuyt, On assessing responsiveness of health-related quality of life instruments: guidelines for instrument evaluation, Qual. Life Res. 12(4) (2003), pp. 349-362.

[21] L. Wiart and J. Darrah, Test-retest reliability of the energy expenditure index in adolescents with cerebral palsy', Dev. Med. Child Neurol. 41(10) (1999), pp. 716-718.

[22] D.A. Winter, The Biomechanics and Motor Control of Human Gait, 2nd ed., University of Waterloo Press, Waterloo, ON, 1990.

[23] F.E. Zajac, R.R. Neptune, and S.A. Kautz, Biomechanics and muscle coordination of human walking. Part I: Introduction to concepts, power transfer, dynamics and simulations, Gait Posture 16(3) (2002), pp. 215-232.

[24] - Biomechanics and muscle coordination of human walking. Part II: Lessons from dynamical simulations and clinical implications, Gait Posture 17(1) (2003), pp. 1-17. 


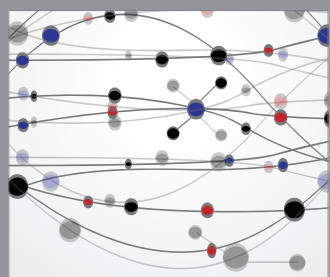

The Scientific World Journal
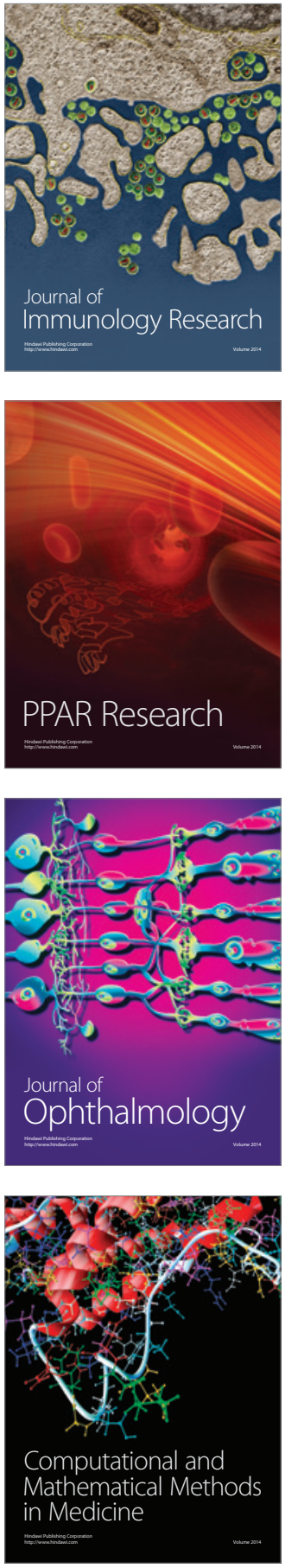

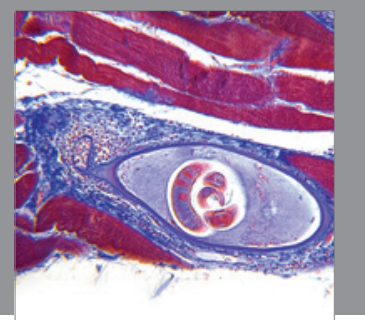

Gastroenterology

Research and Practice
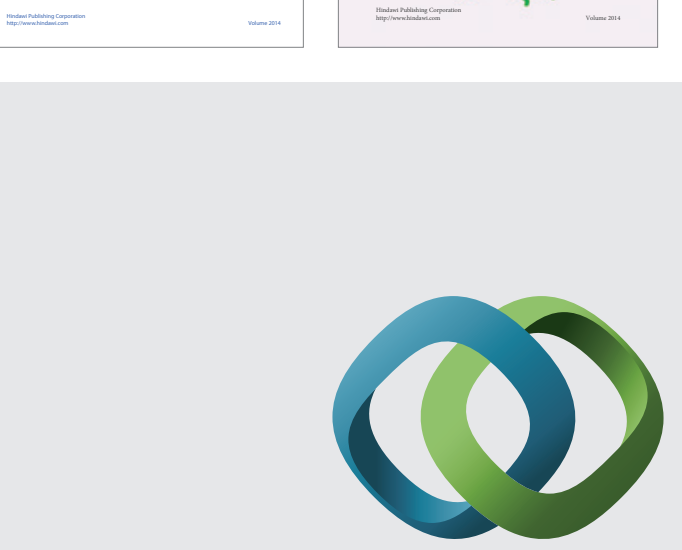

\section{Hindawi}

Submit your manuscripts at

http://www.hindawi.com
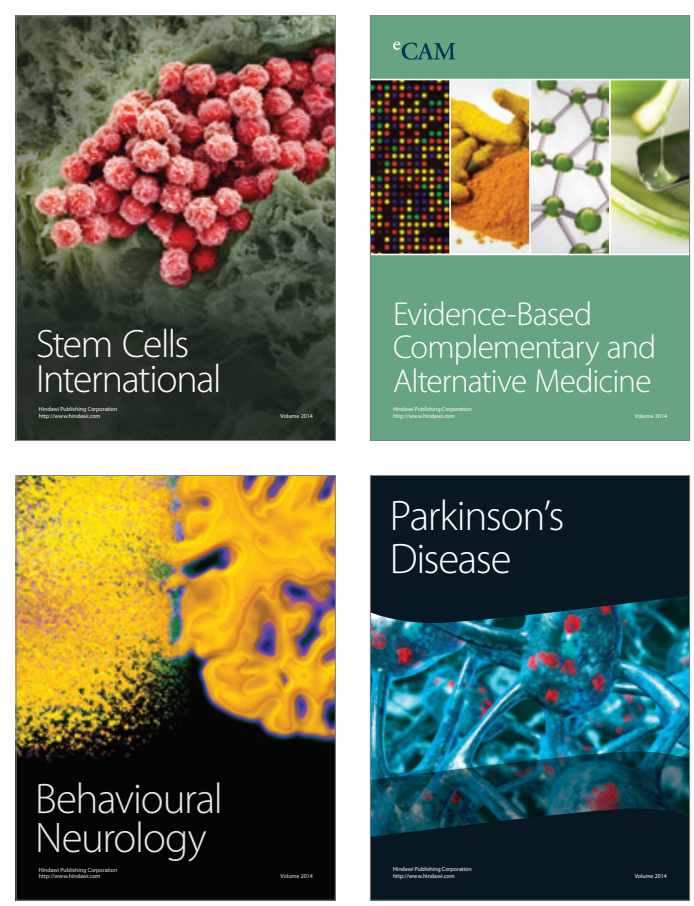

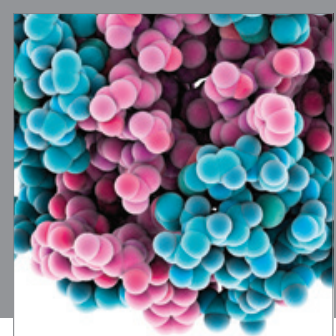

Journal of
Diabetes Research

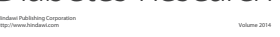

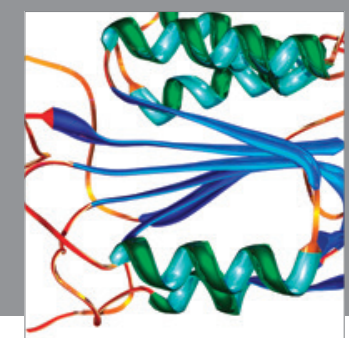

Disease Markers
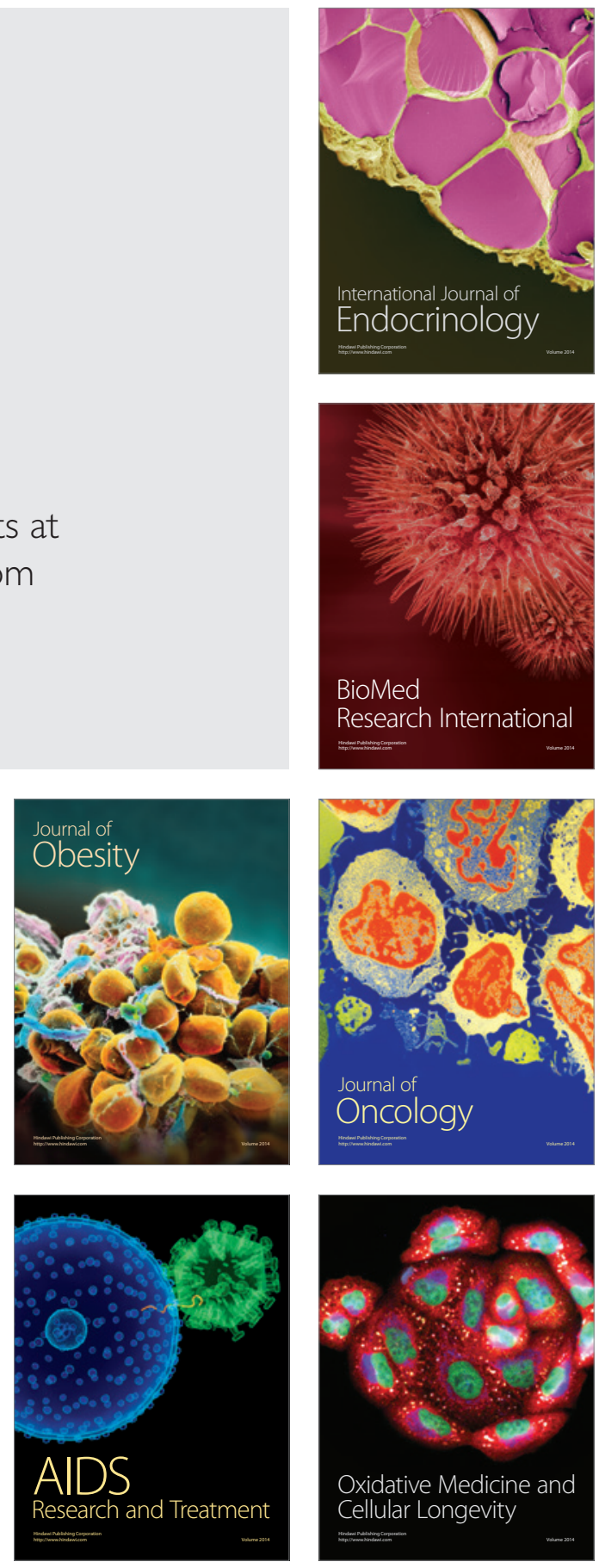